\title{
Construções mentais e distanciamento psicológico: um estudo bibliométrico
}

Leonardo Vils

Professor do MPA-GEAS - Mestrado Profissional em Administração, Gestão Ambiental e Sutentabilidade da Universidade Nove de Julho - UNINOVE vilsleo@gmail.com

Otávio Bandeira De Lamônica Freire

Doutor em Gestão da Comunicação pela USP. Professor Doutor do curso de Marketing da Escola de Artes, Ciências e Humanidades da Universidade de São Paulo, USP

otaviofreire@uninove.br

Marcos Rogério Mazieri

Doutor em Administração de Empresas.Professor do MPA GP -Mestrado Profissional em Gestão de Projetos da Universidade Nove de Julho - UNINOVE

marcosmazzzieri@gmail.com

Gustavo Viegas Rodrigues

Doutor e Mestre pela Universidade Nove de Julho. Professor de cursos de Graduação na Universidade Nove de Julho, UNINOVE

gusviegas@gmail.com

\section{Editor Científico: José Edson Lara}

Organização Comitê Científico

Double Blind Review pelo SEER/OJS

Recebido em 07.08.2018

Aprovado em 24.09.2018

Este trabalho foi licenciado com uma Licença Creative Commons - Atribuição - Não Comercial 3.0 Brasil 


\title{
Resumo
}

Vivemos o aqui e o agora. $\mathrm{O}$ que passou ou acontecerá e o que está distante física, temporal ou socialmente exige que transcendamos o aqui e o agora de maneira a imaginarmos, concebermos ou representarmos aquilo que não é próximo. Da mesma maneira que ao nos afastarmos fisicamente de um objeto perdermos o foco nos detalhes e começamos a notar suas características gerais, o distanciamento psicológico em relação a eventos, pessoas, objetos, localidades e possibilidades muda nossa visão a seu respeito. As distintas representações mentais de eventos próximos e distantes são o foco da Construal Level Theory (CLT). Analisamos, por meio de um estudo bibliométrico, o desenvolvimento da CLT em dois períodos, buscando identificar as origens de teoria, seus desdobramentos e eventual desvinculação de autores e teorias seminais que suportaram o seu desenvolvimento. Os resultados da análise fatorial exploratória das citações nos dois períodos estudados indicam que a CLT se desvincula das teorias seminais que a fundamentaram ganhando corpo teórico próprio.

Palavras-chave: Distanciamento Psicológico, Construal Level Theory, Construções Mentais, Bibliometria.

\section{Mental constructions and psychological distancing: a bibliometric study}

\begin{abstract}
We live here and now. What has happened or will happen and what is physically, temporal, or socially distant requires transcending the here and now in a way that we can imagine, conceive, or represent what is not near. Just as we physically moving away from an object we lose focus on the details and begin to notice its general characteristics the psychological distancing in relation to events, people, objects, locations and possibilities changes our view of them. The distinct mental representations of near and distant events are the focus of the Construal Level Theory (CLT). We analyzed, through a bibliometric study, the development of the CLT in two periods, seeking to identify the origins of theory, its unfolding and eventual untying of authors and seminal theories that supported its development. The results of the exploratory factorial analysis of the citations in the two periods studied indicate that the CLT dissociates itself from the seminal theories that grounded it by gaining its own theoretical body
\end{abstract}

Keywords: Psychological Distance, Construal Level Theory, Mental Construals, Bibliometrics.

\section{Construcciones mentales y distanciamiento psicológico: un estudio bibliométrico}

\section{Resumen}

Vivimos el aquí y el ahora. Lo que pasó o sucederá y lo que está distante física, temporal o socialmente exige que trasciendan el aquí y el ahora de manera a imaginar, concebir o representar lo que no es cercano. De la misma manera que al alejarnos físicamente de un objeto perdemos el foco en los detalles y empezamos a notar sus características generales, el 
distanciamiento psicológico en relación a eventos, personas, objetos, localidades y posibilidades cambia nuestra visión a su respecto. Las distintas representaciones mentales de eventos cercanos y distantes son el foco de la Constricual Level Theory (CLT). Se analizó, por medio de un estudio bibliométrico, el desarrollo de la CLT en dos períodos, buscando identificar los orígenes de teoría, sus desdoblamientos y eventual desvinculación de autores y teorías seminales que soportaron su desarrollo. Los resultados del análisis factorial exploratorio de las citas en los dos períodos estudiados indican que la CLT se desvincula de las teorías seminales que la fundamentaron ganando cuerpo teórico propio.

Palabras clave: Distanciamiento Psicológico, Constricual Level Theory, Construcciones Mentales, Bibliometría.

\section{Introdução}

Escolhas e preferências de um indivíduo a respeito de eventos, objetos ou outros indivíduos podem variar dependendo do momento ou localização em o evento acontece, de sua probabilidade de ocorrência, ou do distanciamento social percebido. Eventos temporal, geográfica, probabilística ou socialmente próximos promovem escolhas diferentes quando comparados a eventos distantes. A perspectiva entre o que é próximo e distante na psicologia cognitiva é conhecida como distanciamento psicológico.

A sua relevância tem implicações teóricas amplas, uma vez que envolve campos da psicologia, comportamento e cognição. A complexidade envolvida na compreensão do fenômeno e a abrangência do tema da distância temporal e sua relação com o nível de construção mental, contextualiza o interesse científico per se, mas há ainda a ausência de consenso entre os autores. A contribuição de Trope e Liberman (2003), apresenta a visão do campo da psicologia, considerando os presupostos da psicodinâmica, notadamente sobre processos primários e secundários, argumentando que a mudança temporal na construção mental, ou seja, quando alteramos o momento em que o evento futuro acontecerá, há mudanças no nível de construção mental e isso está relacionado a mudanças nas escolhas, na predição e nas preferências. Essa contribuição, evidencia que os indivíduos usam modelos mentais mais simples e abstratos para representar a informação de eventos de um futuro distante do que de um futuro próximo.

Tais implicações teóricas e a abrangência do tema em termos de campos de estudos envolvidos, notadamente, a psicologia, o comportamento e a cognição humana, nos estimulou a buscar por uma abordagem bibliométrica, voltada a compreender a dinâmica dos estudos da 
distância temporal relacionada ao nível de construção mental (Construal Level), na literatura científica ao longo das últimas décadas. Nosso objetivo geral é recuperar as publicações científicas publicadas em periódicos de diferentes fatores de impacto e de diferentes épocas, buscando compreender um pouco mais sobre a distância temporal e suas relações com o nível de construção mental, sua evolução, correntes teóricas principais e adjacentes e principais autores. Analisamos as publicações em periódicos científicos em dois períodos, o primeiro, com o objetivo de identificarmos as raízes teóricas do distanciamento psicológico e das construções e/representações mentais, sem limite inferior de datas até o ano de 2009 e o segundo, no intuito de avaliarmos novas contribuições para a teoria, compreende o período entre 2010 e 2016.

Por meio de análise fatorial exploratória encontramos três fatores de agrupamento de autores no primeiro período e dois no segundo. Apresentamos, em seguida, a revisão da literatura, o método de busca e análise das citações, resultados e discussão.

\section{Revisão da Literatura}

Vivemos o aqui e o agora. O que passou ou acontecerá e o que está distante física, temporal ou socialmente exige que transcendamos o aqui e o agora de maneira a imaginarmos, concebermos ou representarmos aquilo que não é próximo. Da mesma maneira que ao nos afastarmos fisicamente de um objeto perdermos o foco nos detalhes e começamos a notar suas características gerais, o distanciamento psicológico em relação a eventos, pessoas, objetos, localidades e possibilidades muda nossa visão a seu respeito. O exemplo de enxergarmos a floresta à distância e as árvores na proximidade - recorrente em artigos sobre CLT (Construal Level Theory) - caracteriza essa relação (Navon, 1977; C. J. Wakslak, Trope, Liberman, \& Alony, 2006). A representação mental de eventos próximos ou distantes tem como ponto de partida o ego. Quanto maior a proximidade de um evento com o ego, maior a sua concretude (Nira Liberman \& Trope, 2008a). Em contrapartida, à medida que um evento se afasta do ego, sua concretude vai sendo substituída paulatinamente por níveis incrementais de abstração. No processo incremental de abstração, características incidentais e contextuais do evento ou objeto acabam por ser omitidas em favor de caracterísicas centrais de maior perenidade. A representação de eventos, assim, de acordo com os pressupostos da CLT, compreende detalhes periféricos, incidentais, contextuais e possivelmente ambíguos quando de sua proximidade e 
por esquemas mentais que extraem do evento suas características centrais, descontextualzadas e livres de ambiguidade quando distantes (N. Liberman, Sagristano, \& Trope, 2002).

A representação de eventos de forma mais concreta ou mais abstrata tem origem na Teoria da Identificação da Ação (Vallacher \& Wegner, 1987, 1989). Uma mesma ação pode ser compreendida de forma mais concreta ou abstrata. Em níveis crescentes de abstração, colocar o dedo em um botão, apertar a campainha e saber há alguém em casa sigficariam a mesma ação. Em termos hierárquicos, colocar o dedo em um botão é a construção de nível baixo e saber se há alguém em casa de nível alto. A CLT incorpora da Teoria da Identificação da Ação o entendimento de ações como de baixo ou alto nível e o instrumento BIF (Behavior Identification Form) desenvolvido para mensuração de características pessoais na categorização mais concreta ou abstrata de eventos. Nesse sentido, representações abstratas são consideradas como de alta construção (high-construal) e as concretas como de baixa construção (low-construal).

Objetos, eventos, objetivos, traços pessoais e ações à medida que se distanciam podem ser representados em hieraquias de abstrações. Um poodle é mais concreto que um cachorro, que, por sua vez, é menos abstrato que um mamífero. Uma mesma ação pode variar de um nível concreto como dar dinheiro, para um menos concreto de ajudar o próximo a um mais abstrato como ser um bom cidadão. Objetivos podem ser representados de forma mais concreta com detalhes de como a tarefa é executada ou mais abstratamente pela razão de sua execução. Grupos socialmente distantes podem ser vistos de forma mais abstrata e homogênea do que grupos socialmente próximos (N. Liberman et al., 2002). O denominador comum às construções mentais concretas ou abstratas é o distanciamento psicológico.

A CLT concebe o distanciamento psicológico em quatro dimensões, temporal, espacial, social e de hipoteticabilidade ou probabilística. Independentemente da dimensão, a construção mental de eventos próximos e distantes segue o mesmo padrão de variação de concreto para abstrato. Locais distantes, longos espaços temporais, pessoas distantes do círculo social e eventos com baixa probabilidade de ocorrência são igualmente percebidos em termos mais abstratos que locais próximos, curtos espaços temporais, pessoas do mesmo círculo social e eventos com alta probabilidade de ocorrência (N. Liberman et al., 2002). Uma vez que as dimensões compartilham o mesmo espectro de representações em relação ao que é próximo, a hipótese de que mudanças em uma dimensão afetam a representação em outras dimensões foi desenvolvida (Nira Liberman \& Forster, 2009). Podemos observar um exemplo de tal influência ao completarmos a frase "Há muito tempo atrás em um lugar " e nossa a tendência em usar "distante” ao invés de "próximo"(Yaacov Trope, Liberman, \& Wakslak, 2007). Em 
que pese a influência de uma dimensão sobre a outra, sua ocorrência só é observada quando a dimensão influenciada é inferida, ou seja, quando não é apresentada. Em uma série de estudos sobre a influência mútua de diferentes dimensões de distanciamento psicológico (Maglio, Trope, \& Liberman, 2013), ao se contrastar duas dimensões foi observada a diminuição da sensibilidade dos participantes ao distanciamento psicológico da segunda dimensão. O estudo concluiu que da mesma forma que uma adição de tempo perde importância na medida que o distanciamento ocorre como, por exemplo, que a distância de hoje a um mes é mais pronunciada do que a distância entre doze e treze meses, a adição a uma dimensão reduz a sensibilidade em outra.

Se, por um lado, o distanciamento psicológico influencia a representação mental de eventos próximos ou distantes por meio de altas ou baixas construções, respectivamente, por outro a relação inversa, do efeito da representação mental no distanciamento também é observada. Representação mental e distanciamento psicológico formam, assim, uma via de mão dupla. A possibilidade de por meio de priming - um estímulo inicial influenciar ass respostas a estímulos subsequentes - interferir no distanciamento psicológico abriu o caminho para testes de hipóteses relacionadas às diferentes dimensões do distanciamento como, por exemplo, o aumento da percepção de distanciamento social pelo uso de palavras e termos mais polidos (Stephan, Liberman, \& Trope, 2010).

Podemos entender os desdobramentos de um acontecimento em julho de 2015 a partir de represntações mentais distintas. Em uma primeira construção: "Um leão foi morto durante uma caçada no Zimbábue." A despeito de nossa preocupação ambiental e para com a vida dos animais, Zimbábue é um país distante; um leão, ainda que mais concreto que um mamífero também é distante; e um caçador é abstrato. Diante da caracterização em termos abstratos do evento tendemos a nos concentrar em seus traços mais centrais, ou seja, em sermos contrários às caçadas e a favor da proteção animal. Passando para uma construção similar à veiculada na imprensa e em redes sociais, temos: "Cecil, o leão símbolo do Zinbábue, foi morto por um dentista americano em uma caçada." O nome do leão, assim como o fato de ser um símbolo, o torna concreto, tornando-o concreto faz com que seja mais próximo. O caçador ganha concretude ao ser identificado como um dentista americano. Não são mais dezenas de leões sem nome que são mortos todos os anos em caçadas na África, é um leão específico, concreto e próximo, praticamente um conhecido. A maldade, mais abstrata, atribuída a caçadores em geral, é, pela identificação concreta do dentista, substituída por assassino nos comentários em 
redes sociais. Assim, a veiculação da notícia em termos mais detalhados como o nome do leão, a profissão do caçador e, posteriormente a arma usada, faz com que interpretemos um acontecimento distante como próximo e que a partir dessa proximidade teçamos nossas considerações a seu respeito. O que dizer então a respeito de mensagens subsequentes nas quais era destacada uma preocupação desmedida com um leão em um país distante enquanto milhares de crianças passam fome no mundo? Milhares de crianças são mais abstratas que centenas de crianças que são menos concretas que dezenas de crianças, mais abstratas que uma criança que, por sua vez, é menos concreta que uma criança com nome. Tomando como base a Lei de Weber Fechner (Fechner, 1966) segundo a qual a capacidade de discernimento entre dois estímulos apresenta uma curva logarítimica em relação à intensidade do estímulo, podemos, independentemente da importância que atribuímos à vida e à redução da fome, nos tornar menos sensíveis à milhares de crianças do que à centenas, dezenas ou a uma e menos ainda do que em relação a uma criança com nome com Alyan Kurdi, que se tornou o símbolo do problema migratório na Europa em 2015. A comoção geral, assim, em termos de CLT, diz menos respeito à indiferença da humanidade para com a fome e a preferência por leões, do que pela maneira como a notícia foi detalhadamente veiculada.

Eventos, objetos e pessoas não são de alta ou baixa construção mental per se. Dois critérios - centralidade e subordinação - identificam características que as tornam de alta ou baixa construção. $\mathrm{O}$ critério da centralidade se baseia no alto impacto gerado pela mudança em uma característica no evento em si. Uma palestra, por exemplo, mudará mais em função de uma mudança no palestrante do que da mudança de sala onde é apresentada (Yaacov Trope \& Liberman, 2010). O critério de subordinação se relaciona à dependência de uma característica em relação à outra. A sala, do exemplo anterior só apresenta alguma importância se o assunto da palestra ou o palestrante despertar interesse. Características de alta ou baixa construção observam resultados diferentes quando distantes. As de alta construção, que apresentam alto valor para o evento, aumentam com a distância e as de baixa construção, de baixo valor para o evento, diminuem. Levando em consideração a valência temos que para as características de alta construção o positivo fica mais positivo e o negativo fica mais negativo com a distância, enquanto para as de baixa construção positivo e negativo são descontados (Yaacov Trope \& Liberman, 2010). Voltando ao exemplo da palestra, um palestrante ou tema que desperte interesse fica mais interessante com a distância enquanto uma sala inapropriada ou desconfortável é vista menos negativamente quando distante. 
O desconto e a amplificação de características têm implicações importantes ao considerarmos as perspectivas teóricas da CLT em relação ao desconto temporal de resultados. Resultados esperados são descontados à medida que se afastam do tempo presente, levando a uma maior preferência por sua antecipação (Loewenstein, 1987). A decisão entre uma alimentação saudável, abstrata, e junk-food para matar a fome, concreta, quando avaliada sob a ótica da CLT estaria vinculada não ao desconto temporal de uma alimentação saudável, mas à comparação entre benefícios abstratos e centrais da alimentação saudável, que aumentam com a distância e a opção em matar a fome aqui e agora, concreta, que aumenta com a proximidade. Some-se à amplificação e desconto de características de alta ou baixa construção os conceitos de desejabilidade e viabilidade (Nira Liberman \& Trope, 2008b), que segundo a teoria se alinham com o distante e o próximo respectivamente, e temos que a dieta saudável é desejada quando distante enquanto a junk-food é viável na proximidade. Nesse sentido, como se compatilizariam a CLT e o auto-controle? Como seria possível fugir do apelo de uma fome concreta em favor de uma saúde abstrata?

O auto-controle é uma decisão em agir de acordo com resultados futuros sacrificando resultados imediatos em seu favor (K. Fujita, Trope, Liberman, \& Levin-Sagi, 2006). Uma vez que resultados futuros são construídos em termos abstratos e que a construção mental e o distanciamento psicológico têm influência recíproca, pensar abstratamente pode ser o caminho para o auto-controle. Construir um evento a partir do porquê é construí-lo abstratamente, enquanto o fazê-lo a partir do como é concreto (Vallacher \& Wegner, 1987). Assim, pensar na razão de uma alimentação saudável em detrimento das dificuldades em segui-la, lembrando que as dificuldades são características subordinadas e sujeitas a serem descontadas com o distanciamento, influencia a decisão de abrir mão de um resultado imediato e favor de um resultado futuro.

A construção mental de eventos próximos ou distantes em termos mais concretos e abstratos, respectivamente, e suas implicações na percepção, categorização e inferência a seu respeito formam a base conceitual da CLT. Construção mental e distanciamento psicológico embora relacionados não se tratam do mesmo conceito, a construção mental ocorre em função do distanciamento e devido à relação recíproca o distanciamento pode ser estimulado por meio de constuções mentais. Construções mentais são, assim, formas de transcender a experiência imediata ou próxima de um evento e de representá-los quando distantes. 


\section{Procedimentos Metodológicos}

Os estudos de citações remontam a doutrina do direito da jurisprudência, ou seja, dos julgamentos em instâncias inferiores serem balizados por aqueles feitos anteriormente em instâncias superiores. Para que atores e decisões anteriores pudessem ser consultados métodos de indexação foram desenvolvidos dando origem às demais metodologias de indexação presentes nos periódicos científicos (Bellis, 2009). O estudo de citações, na medida em que recupera as fontes de consulta e respaldo de autores a respeito de trabalhos anteriores, tornouse um método de mapeamento do desenvolvimento da ciência. Três leis fundamentais, Lotka, Bradford e Zpif caracterizam estudos bibliométricos. A Lei de Lotka prevê que um pequeno número de autores é responsável pela produção de uma grande parcela dos estudos sobre um tema em um determinado campo científico; a Lei de Bradford que uma grande parcela destes estudos é concentrada em um pequeno número de periódicos; e a Lei de Zipf aplica medidas similares às anteriores à recorrência de conceitos e palavras (Bellis, 2009).

Seguindo o protocolo de Quevedo-Silva, Santos, Brandão e Vils (2016), fizemos uma busca em títulos, resumos e palavras-chave no portal de periódicos Web of Sicence com os termos "Construal Level" OR "Psychological Distance". Encontramos 525 artigos compreendendo aproximadamente 30.000 citações. Executamos a mesma busca em dois períodos, até 2010 e a partir de 2011 encontrando 97 e 428 artigos respectivamente. O primeiro período abrange aproximadamente $20 \%$ do total de artigos publicados. Partindo da Lei de Lotka, ordenamos os autores citados em ordem decrescente e selecionamos os $5 \%$ mais citados independentemente do número de citações de cada um. Montamos matrizes de citações, para cada período usando o sofware Bibiexcel e a partir das matrizes fizemos as análises fatoriais exploratórias. A análise fatorial exploratória é uma técnica de redução de dados que se baseia na correlação entre casos ou observações a fatores comuns. Os fatores encontrados representam, assim, em uma menor dimensão, os casos ou observações que o compõem (Quevedo-Silva, Santos, Brandão, \& Vils, 2016). No que diz respeito ao mapeamento de áreas do conhecimento, os fatores indicam a convergência de autores para um determinado tema, assunto ou problema. Os artigos alinhados a cada um dos fatores foram lidos e analisados de forma a nomearmos, apropriadamente, cada um dos fatores encontrados. 


\section{Resultados - Análise Fatorial Exploratória}

Os procedimentos adotados na análise fatorial exploratória seguiram a ordem de análise de KMO (acima de 0,5) de do KMO de cada item na matriz de anti-magem, exclusão de itens com comunalidade abaixo de 0,5 , exclusão de itens com cargas em apenas um fator abaixo de 0,5, exclusão de itens com carga maior em um fator que não o original e exclusão dos itens remanescentes de um fator com confiabilidade (Alpha de Crombach) abaixo de 0,6. Os procedimentos seguem as recomendações de Hair et al. (2009).

A cada extração, com método de componentes principais e rotação Varimax, a análise dos resultados seguiu a ordem acima, e a cada item eliminado nova extração foi executada. Foram retidos, por cumprirem com as exigências de cada etapa de análise, 38 artigos citados no primeiro período (até 2009) e 16 no segundo (a partir de 2010) formando conjuntos de três e dois fatores respectivamente, conforme demonstrado nas Tabelas 1 e 2.

Os resultados das extrações fatoriais nos dois períodos respeitam o critério de KMO maior do que 0,5 (Hair et al., 2009) para cada um dos itens. Os KMO's de 0,922, primeiro período e 0,925 , segundo período, e significância $(\mathrm{p}=000)$ do teste de esfericidade nos dois períodos indicam o ajuste dos itens extraídos à análise fatorial.

No primeiro período os três fatores explicam $69,26 \%$ da variância total e os dois fatores do segundo período explicam $78,46 \%$, resultados alinhados com as recomendações da literatura (Hair et al., 2009).

\section{Tabela 2}

Análise Fatorial Exploratória - A partir de 2010

\begin{tabular}{|l|r|r|}
\hline Matriz de Componentes Rotacionada & \multicolumn{2}{|c|}{ Componente } \\
\cline { 2 - 3 } Citação & Consolidação & Validação \\
\hline (K. Fujita, Trope, Liberman, \& Levin-Sagi, 2006a) &, 843 & \\
(Kentaro Fujita, Eyal, Chaiken, Trope, \& Liberman, &, 826 & \\
2008) &, 790 & \\
(Todorov, Goren, \& Trope, 2007) &, 774 & \\
(Liviatan, Trope, \& Liberman, 2008) &, 761 & \\
(Sagristano, Trope, \& Liberman, 2002) &, 747 & \\
(C. J. Wakslak et al., 2006) &, 716 & \\
(Higgins, 1997) & &, 892 \\
(Yaacov Trope et al., 2007) & &, 873 \\
\hline (N. Liberman, Sagristano, \& Trope, 2002) & \\
(N. Liberman \& Trope, 1998) & \\
(Nira Liberman, Trope, \& Wakslak, 2007) &, 783 \\
(Kentaro Fujita \& Han, 2009) & &, 725 \\
(Nira Liberman \& Förster, 2009) &, 674 \\
(Aiken, 1991) & &
\end{tabular}




\begin{tabular}{|l|r|r|} 
(Baron \& Kenny, 1986) & &, 636 \\
\hline Alpha de Cronbach & 0,912 & 0,884 \\
\hline Variânca \% Explicada - Cumulativa & 70,11 & 78,46 \\
\hline
\end{tabular}

Elaborada pelos autores

\section{Discussão}

O primeiro fator encontrado no período até 2010, responsável por 53,9\% da variância explicada, diz respeito aos testes empíricos das quatro dimensões do distanciamento psicológico, espacial, temporal, social e de hipoteticabilidade. Nesse período inicial do desenvolvimento teórico, a Teoria da Identificação da Ação (Vallacher \& Wegner, 1987) e o instrumento BIF (Behavior Identification Form) (Vallacher \& Wegner, 1989) oferecem tanto o embasamento para a definição de representações da alta e baixa construção quanto para sua mensuração. A Teoria da Identificação da Ação propõe que que ações podem ser identificadas de diversas maneiras, variando de identidades de nível baixo, que se baseiam em como uma ação é executada, a identidades de nível alto, baseadas na razão pela qual a ação foi executada (Vallacher \& Wegner, 1987). O instrumento BIF (Behavior Identification Form) busca, por meio da contraposição de definições de baixo e alto nível, encontrar características de personalidade que, em linhas gerais, definam a predisposição a agentes de nível baixo ou de nível alto (Vallacher \& Wegner, 1989). Agentes de nível alto entendem suas ações em termos da motivação para a sua execução e significados amplos e os de nível baixo em termos de como executá-las e significados detalhados (Vallacher \& Wegner, 1987). O instrumento BIF foi usado basicamente como variável dependente nos estudos experimentais como medida do nível de construção mental por meio do alinhamento das identificações de nível baixo com as construções mentais de nível baixo e das identificações de nível alto com construções mentais de nível alto. A CLT parte da Teoria da Identificação da Ação, incorporando as identidades de nível alto e baixo e as renomeando como construções mentais de nível alto e baixo, e se diferenciando no sentido que as construções mentais ou identidades não são necessariamente características psicológicas, mas dependentes do distanciamento psicológico.

A primeira aplicação da relação entre distanciamento psicológico e construções mentais baseou-se nos conceitos de desejabilidade e viabilidade na consecução de objetivos. Objetivos distantes foram caracterizados pelo desejo de vê-los completos e os mais próximos pela viabilidade de concluí-los (N. Liberman \& Trope, 1998). Posteriormente, a desejabilidade de estados futuros foi usada como estímulo para autocontrole que, segundo a CLT, se configura 
em abrir mão de um estado presente concreto e viável, em favor de um estado futuro desejado (K. Fujita et al., 2006).

A relação entre o distanciamento temporal e as construções mentais foi complementada pela definição de atributos de nível alto e de nível baixo e as implicações de suas valências. Atributos de nível alto são aqueles cuja mudança tem impacto significativo em um evento sendo definidos por sua centralidade. Atributos de nível baixo, por sua vez, são subordinados aos de nível alto e uma mudança não impacta significativamente em um evento. Independentemente de sua valência, positiva ou negativa, atributos de nível alto são amplificados com a distância e os de nível baixo descontados (Y. Trope \& Liberman, 2000). Nesse sentido, a CLT amplia o entendimento de escolhas intertemporais (Loewenstein, 1987). Estados ou eventos futuros não são simplesmente descontados, mas dependentes do valor ou nível de seus atributos.

A ativação de construções mentais abstratas, de nível alto, e concretas, de nível baixo, em uma dimensão e sua influência em outras dimensões está presente nos artigos alinhados a este fator. Dimensões espaciais mais distantes implicam na percepção de distanciamento social (Henderson, Fujita, Trope, \& Liberman, 2006) e estímulos de poder ao evocarem construções mentais abstratas aumentam o distanciamento psicológico entre pessoas (Smith \& Trope, 2006). Independentemente da dimensão, o distanciamento psicológico se associa a palavras ou conceitos mais abstratos para eventos mais distantes, e a palavras ou conceitos mais concretos para eventos mais próximos (Bar-Anan, Liberman, \& Trope, 2006). A transição da experiência vivida, aqui e agora, para a imaginada, distante, e o caminho inverso dependem, assim, de construções mentais congruentes com o distanciamento psicológico.

Um outro instrumento de uso recorrente nos experimentos de distanciamento é o Navon Test que compreende a formação de letras por meio de outras letras menores, Figura1(esquerda), (Navon, 1977). Em termos da CLT, construções de nível alto, distantes são relacionadas às características centrais de um evento - a letra $\mathrm{T}$ da figura - enquanto as construções de nível baixo, próximas, são relacionadas ao detalhe - as letras S. Construções abstratas permitem a compreensão de figuras da Gestalt, Figura 1(direita), e as concretas a inibem havendo assim uma relação entre níveis de construções mentais e a criatividade (Forster, Friedman, \& Liberman, 2004) 

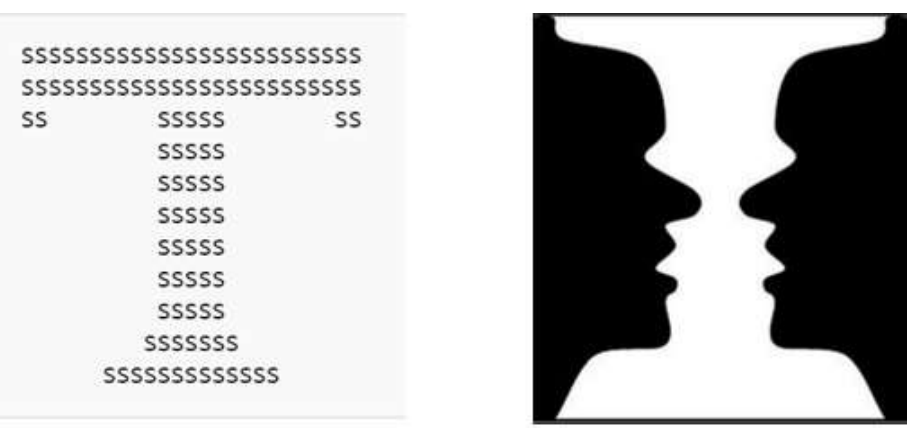

Figura 1 - Navon Test e Gestalt

Dois outros artigos se destacam neste fator. O primeiro relacionando o distanciamento psicológico à Teoria do Foco Regulatório (Higgins, 1997), encontrando a associação entre objetivos distantes e o foco da promoção (Pennington \& Roese, 2003), e o segundo associando o distanciamento às características centrais de produtos (Lynch \& Zauberman, 2007).

Artigos alinhados ao primeiro fator, cujas contribuições estão centradas nos testes hipóteses da CLT de distanciamento psicológico e construções mentais concretas e abstratas os caracterizam como aqueles que deram origem ao desenvolvimento da teoria. Nomeamos, assim, o primeiro fator como "Origens".

Em que pese a variância explicada de $10,85 \%$, praticamente um quinto do primeiro fator, o segundo fator apresenta as contribuições de teorias anteriores às percepções e julgamento segundo o distanciamento social, ao autocontrole, à memória e aos sistemas de processamento mental. A Teoria da Atribuição (Heider, 1958) prevê que a percepção social, tal qual a percepção de objetos, está sujeita a vieses e ilusões cognitivas, razão pela qual tendemos a ancorar o julgamento de outros em suas características de personalidade e atitudes omitindo influências contextuais e situacionais. Estes vieses dão origem ao Erro Fundamental de Atribuição. Atribuímos à personalidade e à atitude dos outros aquilo que poderia ser contextual (D. Gilbert \& Malone, 1995) por meio de adjetivos abstratos, e a nós questões situacionais por meio de verbos concretos (Fiedler, Semin, Finkenauer, \& Berkel, 1995). Com relação ao julgamento e percepções de grupos sociais, vemos os grupos a que não pertencemos como mais homogêneos e aqueles em que estamos inseridos com mais variabilidade; os outros são todos iguais e nós somos diferentes (Linville, Fischer, \& Yoon, 1996).

O experimento do marshmallow (Mischel, Shoda, \& Rodriguez, 1989) é, provavelmente, a referência mais conhecida fora do ambiente acadêmico acerca do autocontrole e do adiamento da gratificação. Escolher entre um marshmallow agora, concreto, por meio de um sistema cognitivo intuitivo ou quente, ou dois depois, mais abstrato, fazendo uso de um sistema cognitivo mais frio e racional (Metcalfe \& Mischel, 1999), está em linha com os 
desenvolvimentos posteriores da CLT segundo a qual o pensamento abstrato influencia positivamente o autocontrole. Eventos sociais passados armazenados na memória ganham maiores graus de abstração com o distanciamento, sendo representados por linguagem mais concreta conforme a proximidade (Semin \& Smith, 1999). A CLT incorpora essas representações a as relaciona também com o distanciamento futuro. Os artigos alinhados ao segundo fator formam referências básicas para o desenvolvimento da CLT, assim, nomeamos o fator como "Clássicos".

O terceiro fator, ainda que com baixa contribuição para o total da variância explicada, também é formado por artigos que alavancaram o desenvolvimento da CLT. O fator inclui artigos relacionados aos estados de humor e atribuição (Schwarz \& Clore, 1983), estados de humor e afeto (Strack, Schwarz, \& Gschneidinger, 1985), consecução de objetivos (Buehler, Griffin, \& Ross, 1994; Gilovich, Kerr, \& Medvec, 1993), otimismo (Shepperd, Ouellette, \& Fernandez, 1996) e dificuldade em estimar estados afetivos futuros (D. T. Gilbert, Pinel, Wilson, Blumberg, \& Wheatley, 1998). Também estão presentes no fator artigos e livros referentes aos métodos estatísticos (Kenny, 1998; Sobel 1982). Nomeamos o terceiro fator como "Complementares".

No segundo período de análise, a partir do ano de 2011, dois fatores, representando $70,11 \%$ e $8,35 \%$ da variância explicada foram encontrados. Nota-se, no período, que os artigos clássicos que fundamentaram o desenvolvimento da teoria não mais aparecem entre os mais citados indicando a consolidação da teoria por meio da citação de seus autores mais relevantes, Liberman e Trope (N. Liberman \& Trope, 1998). O primeiro fator apresenta artigos relacionados ao aprofundamento dos testes de hipóteses com ênfase na probabilidade (ou hipoteticabilidade) (Sagristano, Trope, \& Liberman, 2002; Todorov, Goren, \& Trope, 2007; C. Wakslak \& Trope, 2009), autocontrole (K. Fujita et al., 2006), integração com a Teoria do Foco Regulatório (Higgins, 1997), e julgamento interpessoal (Liviatan, Trope, \& Liberman, 2008). Uma vez que autores seminais e teorias clássicas que fundamentaram o desenvolvimento da CLT deixam de ser citados e são substituídos por aqueles que a desenvolveram, podemos inferir que a CLT se consolida e ganha corpo teórico próprio. Nomeamos, assim, o primeiro fator do segundo período de estudo como "Consolidação".

O segundo fator, com baixa contribuição para a variância explicada, refere-se a artigos que reforçam e validam os resultados encontrados em estudos anteriores a respeito de 
autocontrole (Kentaro Fujita \& Han, 2009), categorização (N. Liberman et al., 2002) e processamento mental (Nira Liberman \& Förster, 2009). Nomeamos o fator como "Validação".

Os resultados da análise fatorial exploratória nos dois períodos estudados indicam o primeiro período como o desenvolvimento da CLT baseado em teorias psicológicas já estabelecidas, notadamente a Teoria da Identificação da Ação (Vallacher \& Wegner, 1987) e um segundo período no qual Nira Liberman e Yacoov Trope se consolidam como referências principais da CLT.

\section{Conclusão}

Mapeamos, por meio da análise de citações, 525 artigos publicados em periódicos acadêmicos a respeito do distanciamento psicológico e da Contrual Level Theory. Nosso objetivo central nesse artigo era de apresentar os conceitos fundamentais da teoria, os principais autores e seu desenvolvimento. Ao separarmos as citações em dois períodos pudemos observar a importância de autores seminais, clássicos, ao desenvolvimento do corpo teórico e, assim, contribuímos para uma maior compreensão sobre os fundamentos da teoria. Identificamos também o alinhamento de autores e artigos em fatores contribuindo para o entendimento do desenvolvimento da teoria. Percebe-se claramente o momento de desenvolvimento e referenciação dos estudos seminais, bem como a configuração dos expoentes da teoria na primeira fase, enquanto que, na segunda fase, a CLT se consolida e os estudos e autores a partir de 2011 buscam a sua solidificação. Estamos cientes das limitações de um estudo bibliométrico. Há autores e artigos pouco citados que podem estar apresentando novas perspectivas para o campo e há, provavelmente, outras áreas da ciência que poderiam incluídas na busca e poderíamos também ter trabalhado com um foco mais estreito como Business Sciences, entretanto, julgamos o escopo adequado para um trabalho exploratório sobre as origens, conceitos e desenvolvimento da Construal Level Theory.

\section{Referências}

Araújo, C. A. A. (2006). Bibliometria: evolução histórica e questões atuais. Em Questão, 12(1). https://doi.org/10.19132/1808-5245121.

Arima, A. N. (2003). Gender Stereotypes in Japanese Television Advertisements. Sex Roles, 49(1-2), 81-90. https://doi.org/10.1023/A:1023965704387 
Asano-Cavanagh, Y. (2014). Linguistic manifestation of gender reinforcement through the use of the Japanese term kawaii. Gender and Language, 8(3). https://doi.org/10.1558/genl.v8i3.341

Bargh, J. A., Chen, M., \& Burrows, L. (1996). Automaticity of social behavior: Direct effects of trait construct and stereotype activation on action. Journal of Personality and Social Psychology, 71(2), 230-244. https://doi.org/10.1037/0022-3514.71.2.230

Bargh, J. A., Gollwitzer, P. M., Lee-Chai, A., Barndollar, K., \& Trötschel, R. (2001). The automated will: Nonconscious activation and pursuit of behavioral goals. Journal of Personality and Social Psychology, 81(6), 1014-1027. https://doi.org/10.1037/00223514.81.6.1014

Bartsch, R. A., Burnett, T., Diller, T. R., \& Rankin-Williams, E. (2000). Gender Representation in Television Commercials: Updating an Update. Sex Roles, 43(9-10), 735-743. https://doi.org/10.1023/A:1007112826569

Bettman, J. R., \& Sujan, M. (1987). Effects of Framing on Evaluation of Comparable and Noncomparable Alternatives by Expert and Novice Consumers. Journal of Consumer Research, 14(2), 141-154. https://doi.org/10.1086/209102

Boyack, K. W., Wylie, B. N., \& Davidson, G. S. (2002). Domain visualization using VxInsight ${ }^{\circledR}$ for science and technology management. Journal of the American Society for Information Science and Technology, 53(9), 764-774. https://doi.org/10.1002/asi.10066

Bresnahan, M. J., Inoue, Y., Liu, W. Y., \& Nishida, T. (2001). Changing Gender Roles in Prime-Time Commercials in Malaysia, Japan, Taiwan, and the United States. Sex Roles, 45(1-2), 117-131. https://doi.org/10.1023/A:1013068519583

Bretl, D. J., \& Cantor, J. (1988). The portrayal of men and women in U.S. television commercials: A recent content analysis and trends over 15 years. Sex Roles, 18(9-10), 595609. https://doi.org/10.1007/BF00287963

Chung, K. H., \& Cox, R. A. K. (1990). Patterns of Productivity in the Finance Literature: A Study of the Bibliometric Distributions. The Journal of Finance, 45(1), 301-309. https://doi.org/10.2307/2328824

Coltrane, S., \& Messineo, M. (2000). The Perpetuation of Subtle Prejudice: Race and Gender Imagery in 1990s Television Advertising. Sex Roles, 42(5-6), 363-389. https://doi.org/10.1023/A:1007046204478

Courtney, A. E., \& Whipple, T. W. (1983). Sex stereotyping in advertising. Lexington Books.

Craig, R. S. (1992a). The effect of television day part on gender portrayals in television commercials: A content analysis. Sex Roles, 26(5-6), 197-211. https://doi.org/10.1007/BF00289707

Craig, R. S. (1992b). The effect of television day part on gender portrayals in television commercials: A content analysis. Sex Roles, 26(5-6), 197-211. https://doi.org/10.1007/BF00289707

Dijksterhuis, A., Smith, P. K., van Baaren, R. B., \& J, H. (2005). The Unconscious Consumer: Effects of Environment on Consumer Behavior. Journal of Consumer Psychology, 15(3), 193-202. https://doi.org/10.1207/s15327663jcp1503_3

Ferguson, M. J., \& Bargh, J. A. (2004). Liking Is for Doing: The Effects of Goal Pursuit on Automatic Evaluation. Journal of Personality and Social Psychology, 87(5), 557-572. https://doi.org/10.1037/0022-3514.87.5.557

Furnham, A., \& Mak, T. (1999). Sex-Role Stereotyping in Television Commercials: A Review and Comparison of Fourteen Studies Done on Five Continents Over 25 Years. Sex Roles, 41(5-6), 413-437. https://doi.org/10.1023/A:1018826900972 
Furnham, A., \& Paltzer, S. (2010). The portrayal of men and women in television advertisements: An updated review of 30 studies published since 2000. Scandinavian Journal of Psychology, 51(3), 216-236. https://doi.org/10.1111/j.1467-9450.2009.00772.x

Furnham, A., \& Steele, H. (1993). Measuring locus of control: a critique of general, children's, health- and work-related locus of control questionnaires. British Journal of Psychology (London, England: 1953), 84 ( Pt 4), 443-479.

Ganahl, D. J., Prinsen, T. J., \& Netzley, S. B. (2003). A Content Analysis of Prime Time Commercials: A Contextual Framework of Gender Representation. Sex Roles, 49(9-10), 545-551. https://doi.org/10.1023/A:1025893025658

Garst, J., \& Bodenhausen, G. V. (1997). Advertising's Effects on Men's Gender Role Attitudes. Sex Roles, 36(9-10), 551-572. https://doi.org/10.1023/A:1025661806947

Gerbner, G., Gross, L., Signorielli, N., \& Morgan, M. (1980). Aging with Television: Images on Television Drama and Conceptions of Social Reality. Journal of Communication, 30(1), 37-47. https://doi.org/10.1111/j.1460-2466.1980.tb01766.x

Goldberg, M. E., \& Gorn, G. J. (1987). Happy and Sad TV Programs: How They Affect Reactions to Commercials. Journal of Consumer Research, 14(3), 387-403.

Gollwitzer, P. M. (1999). Implementation intentions: Strong effects of simple plans. American Psychologist, 54(7), 493-503. https://doi.org/10.1037/0003-066X.54.7.493

Hair, J. F., BLACK, Anderson, R. E., \& Tatham, R. L. (2005). Analise Multivariada de Dados. Bookman.

Hawkins, D. (1970). The Effects of Subliminal Stimulation on Drive Level and Brand Preference. Journal of Marketing Research, 7(3), 322-326. https://doi.org/10.2307/3150289

Herr, P. M. (1989). Priming Price: Prior Knowledge and Context Effects. Journal of Consumer Research, 16(1), 67-75. https://doi.org/10.1086/209194

Kunst-Wilson, W. R., \& Zajonc, R. B. (1980). Affective discrimination of stimuli that cannot be recognized. Science (New York, N.Y.), 207(4430), 557-558.

Markus, H. R., \& Kitayama, S. (1991). Culture and the self: Implications for cognition, emotion, and motivation. Psychological Review, 98(2), 224-253. https://doi.org/10.1037/0033-295X.98.2.224

Mastro, D. E., \& Stern, S. R. (2003). Representations of Race in Television Commercials: A Content Analysis of Prime-Time Advertising. Journal of Broadcasting \& Electronic Media, 47(4), 638-647. https://doi.org/10.1207/s15506878jobem4704_9

Mazzella, C., Durkin, K., Cerini, E., \& Buralli, P. (1992). Sex role stereotyping in Australian television advertisements. Sex Roles, 26(7-8), 243-259. https://doi.org/10.1007/BF00289910

Milner, L. M. (2005). Sex-Role Portrayals in African Television Advertising. Journal of International Consumer Marketing, 17(2-3), 73-91. https://doi.org/10.1300/J046v17n02_05

Monica M. Lee, B. C. (2007). Representations of older adults in television advertisements. Journal of Aging Studies, 21(1), 23-30. https://doi.org/10.1016/j.jaging.2006.04.001

Moore, R. S., Stammerjohan, C. A., \& Coulter, R. A. (2005). Banner advertiser-web site context congruity and color effects on attention and attitudes. Journal of advertising, 34(2), 71-84.

Mwangi, M. W. (1996). Gender roles portrayed in Kenyan television commercials. Sex Roles, 34(3-4), 205-214. https://doi.org/10.1007/BF01544296

Nassif, A., \& Gunter, B. (2008). Gender Representation in Television Advertisements in Britain and Saudi Arabia. Sex Roles, 58(11-12), 752-760. https://doi.org/10.1007/s11199-0089394-6 
Nedungadi, P. (1990). Recall and Consumer Consideration Sets: Influencing Choice without Altering Brand Evaluations. Journal of Consumer Research, 17(3), 263-276. https://doi.org/10.1086/208556

Pereira Câmara Leal, R., de Oliveira, J., \& Feldman Soluri, A. (2003). Perfil da pesquisa em finanças no Brasil. Revista de Administração de Empresas, 43(1), 1-14. https://doi.org/10.1590/S0034-75902003000100010

Perreault, W. D., \& Leigh, L. E. (1989). Reliability of Nominal Data Based on Qualitative Judgments. Journal of Marketing Research, 26(2), 135-148. https://doi.org/10.2307/3172601

Petrides, K. V., \& Furnham, A. (2000). Gender Differences in Measured and Self-Estimated Trait Emotional Intelligence. Sex Roles, 42(5-6), 449-461. https://doi.org/10.1023/A:1007006523133

Petrides, K. V., \& Furnham, A. (2001). Trait emotional intelligence: psychometric investigation with reference to established trait taxonomies. European Journal of Personality, 15(6), 425448. https://doi.org/10.1002/per.416

Petty, R. E., Cacioppo, J. T., \& Schumann, D. (1983). Central and Peripheral Routes to Advertising Effectiveness: The Moderating Role of Involvement. Journal of Consumer Research, 10(2), 135. https://doi.org/10.1086/208954

Ph.D, F. S., \& Ph.D, Q. C. (2007). Contextual Priming and Applicability: Implications for Ad Attitude and Brand Evaluations. Journal of Advertising, 36(1), 69-80. https://doi.org/10.2753/JOA0091-3367360105

Ph.D, L. M. M., \& [honours, B. H. B. A. (2004). Gender Sex-Role Portrayals in International Television Advertising over Time: The Australian Experience. Journal of Current Issues \& Research in Advertising, 26(2), 81-95. https://doi.org/10.1080/10641734.2004.10505166

Pollay, R. W. (1986). The Distorted Mirror: Reflections on the Unintended Consequences of Advertising. Journal of Marketing, 50(2). Recuperado de https://works.bepress.com/richard_pollay/30/

Pritchard, A. (1969). Statistical Bibliography or Bibliometrics? Journal of Documentation, 25(4), 348-349.

Quevedo-Silva, F., Santos, E. B. A., Brandão, M. M., \& Vils, L. (2016). Estudo Bibliométrico: Orientações sobre sua Aplicação. Revista Brasileira de Marketing e-ISSN:2177-5184, 15(2), 246-262.

Richard E. Petty, J. T. C. (1986). The Elaboration Likelihood Model of Persuasion. Advances in Experimental Social Psychology, 19, 123-205. https://doi.org/10.1016/S00652601(08)60214-2

Russell, C. A. (2002). Investigating the Effectiveness of Product Placements in Television Shows: The Role of Modality and Plot Connection Congruence on Brand Memory and Attitude. Journal of Consumer Research, 29(3), 306-318. https://doi.org/10.1086/344432

Santos, R. N. M. dos, \& Kobashi, N. Y. (2012). Bibliometria, Cientometria, Infometria: Conceitos e Aplicações. Pesquisa Brasileira em Ciência da Informação e Biblioteconomia, 5(1). Recuperado de http://periodicos.ufpb.br/ojs/index.php/pbcib/article/view/11992

Schmitt, B. H. (1994). Contextual priming of visual information in advertisements. Psychology and Marketing, 11(1), 1-14. https://doi.org/10.1002/mar.4220110103

Schwarz, N., \& Clore, G. L. (1983). Mood, misattribution, and judgments of well-being: Informative and directive functions of affective states. Journal of Personality and Social Psychology, 45(3), 513-523. https://doi.org/10.1037/0022-3514.45.3.513 
Siu, W., \& Kai-ming Au, A. (1997). Women in advertising: a comparison of television advertisements in China and Singapore. Marketing Intelligence \& Planning, 15(5), 235-243. https://doi.org/10.1108/02634509710177305

Soldow, G. F., \& Principe, V. (1981). Response to commercials as a function of program context. Journal of Advertising Research, 21(2), 59-65.

Subramanyam, K. (1983). Bibliometric studies of research collaboration: A review. Journal of Information Science, 6(1), 33-38. https://doi.org/10.1177/016555158300600105

Tan, T. T. W., Ling, L. B., \& Theng, E. P. C. (2002). Gender-role portrayals in Malaysian and Singaporean television commercials: an international advertising perspective. Journal of Business Research, 55(10), 853-861. https://doi.org/10.1016/S0148-2963(00)00225-3

Tulving, E., \& Schacter, D. L. (1990). Priming and human memory systems. Science, 247(4940), 301-306. https://doi.org/10.1126/science.2296719

Uray, N., \& Burnaz, S. (2003). An Analysis of the Portrayal of Gender Roles in Turkish Television Advertisements. Sex Roles, 48(1-2), 77-87. https://doi.org/10.1023/A:1022348813469

Valls-Fernández, F., \& Martínez-Vicente, J. M. (2007). Gender Stereotypes in Spanish Television Commercials. Sex Roles, 56(9-10), 691-699. https://doi.org/10.1007/s11199007-9208-2

Yang, M., \& Roskos-Ewoldsen, D. R. (2007). The Effectiveness of Brand Placements in the Movies: Levels of Placements, Explicit and Implicit Memory, and Brand-Choice Behavior. Journal of Communication, 57(3), 469-489. https://doi.org/10.1111/j.14602466.2007.00353.x

Zajonc, R. B. (1968). Attitudinal Effects of Mere Exposure. Journal of Personality and Social Psychology, 9(2, Pt.2), 1-27. https://doi.org/10.1037/h0025848 